\title{
Spin waves and the origin of commensurate magnetism in $\mathrm{Ba}_{2} \mathrm{CoGe}_{2} \mathrm{O}_{7}$
}

\author{
A. Zheludev* \\ Condensed Matter Sciences Division, Oak Ridge National Laboratory, Oak Ridge, Tennessee 37831-6393, USA
}

T. Sato, T. Masuda, ${ }^{\dagger}$ and K. Uchinokura

Department of Advanced Materials Science, The University of Tokyo, Tokyo 113-8656, Japan

G. Shirane

Physics Department, Brookhaven National Laboratory, Upton, New York 11973-5000, USA

B. Roessli

Laboratory for Neutron Scattering, ETH Zurich and Paul Scherrer Institute, CH-5232 Villigen PSI, Switzerland

(Received 27 February 2003; revised manuscript received 8 May 2003; published 30 July 2003)

\begin{abstract}
The square-lattice antiferromagnet $\mathrm{Ba}_{2} \mathrm{CoGe}_{2} \mathrm{O}_{7}$ is studied by means of neutron diffraction and inelastic scattering. This material is isostructural to the well-known Dzyaloshinskii-Moriya helimagnet $\mathrm{Ba}_{2} \mathrm{CuGe}_{2} \mathrm{O}_{7}$ but exhibits commensurate long-range Néel order at low temperatures. Measurements of the spin wave dispersion relation reveal strong in-plane anisotropy that is the likely reason for the suppression of helimagnetism.
\end{abstract}

DOI: $10.1103 /$ PhysRevB.68.024428

PACS number(s): 75.30.Ds, 75.25.+z, 75.50.Ee

\section{INTRODUCTION}

Several years ago, the square-lattice helimagnet $\mathrm{Ba}_{2} \mathrm{CuGe}_{2} \mathrm{O}_{7}$ was recognized as an extremely interesting material for studying Dzyaloshinskii-Moriya (DM) offdiagonal exchange interactions. Thanks to its low (tetragonal) crystal symmetry and convenient magnetic energy scales, it is prototype for experimental studies of several phenomena, not accessible in such well-known cubic helimagnets as $\mathrm{FeGe}^{1}$ and $\mathrm{MnSi}^{2}$ In particular, $\mathrm{Ba}_{2} \mathrm{CuGe}_{2} \mathrm{O}_{7}$ sports a unique field-induced incommensurate-to-commensurate (IC) transition, ${ }^{3-5}$ and an interesting field dependence of the spiral spin structure. ${ }^{6}$ This material also enabled the first direct observation of a new type of magnetic interactions, namely, the so-called Kaplan anisotropy, that in most cases is a companion to DM interactions. ${ }^{9-13}$ Additional theoretical work shed light on the nature of the complex "intermediate phase." ${ }^{14}$ A general theoretical description of noncentrosymmetric tetragonal antiferromagnets ${ }^{15}$ recently placed $\mathrm{Ba}_{2} \mathrm{CuGe}_{2} \mathrm{O}_{7}$ in one category with another new and very interesting material, namely, the weak-ferromagnet $\mathrm{K}_{2} \mathrm{~V}_{3} \mathrm{O}_{8} .{ }^{16}$

A recent study indicated that an IC transition in $\mathrm{Ba}_{2} \mathrm{CuGe}_{2} \mathrm{O}_{7}$ can be induced not only by applying an external magnetic field, but also by a partial chemical substitution of the spin-carrying $\mathrm{Cu}^{2+}$ ions by $\mathrm{Co}^{2+} \cdot{ }^{17}$ For all Co-concentrations $x$, the solid solution $\mathrm{Ba}_{2}\left(\mathrm{Co}_{x} \mathrm{Cu}_{1-x}\right) \mathrm{Ge}_{2} \mathrm{O}_{7}$ orders magnetically at temperatures between $T_{N}=3.2 \mathrm{~K}(x=0)$ and $T_{N}=6.7 \mathrm{~K}(x=1)$. Magnetization data suggest that the helimagnetic state realized at $x=0$ gives way to a canted weak-ferromagnetic structure at some critical concentration $x_{c}$, estimated to be between 0.05 and 0.1. The mechanism of this transition or crossover is poorly understood. One possible explanation was proposed in Ref. 17. The structure of $\mathrm{Ba}_{2}\left(\mathrm{Co}_{x} \mathrm{Cu}_{1-x}\right) \mathrm{Ge}_{2} \mathrm{O}_{7}$ is tetragonal, the $S=1 / 2 \mathrm{Cu}^{2+}$ or $S=3 / 2 \mathrm{Co}^{2+}$ ions forming a square lattice within the $(a, b)$ crystallographic plane. The dominant interaction is the antiferromagnetic (AF) coupling $J$ between nearest-neighbor $(\mathrm{NN})$ sites along the $(1,1,0)$ direction. In the $x=0$ compound, the helimagnetic distortion is caused by the in-plane component $\mathbf{D}_{x y}$ of the Dzyaloshinskii vector $\mathbf{D}$ associated with the same $\mathrm{Cu}-\mathrm{Cu}$ bonds. ${ }^{4,5}$ This component retains its direction from one bond to the next and thus, favors a spin spiral state. ${ }^{18}$ In contrast, the out-of-plane component $\mathbf{D}_{z}$ is sign-alternating and stabilizes a weakferromagnetic structure. The $z$-axis component of $\mathbf{D}$ was never detected in $\mathrm{Ba}_{2} \mathrm{CuGe}_{2} \mathrm{O}_{7}$, where it was assumed to be weak. In Ref. 17, it was tentatively suggested that the outof-plane component is dominant in the Co-based $x=1$ material and stabilizes a commensurate magnetic structure. To verify this hypothesis and better understand the underlying physics, a detailed knowledge of magnetic interactions not only in $\mathrm{Ba}_{2} \mathrm{CuGe}_{2} \mathrm{O}_{7}(x=0)$, but also in $\mathrm{Ba}_{2} \mathrm{CoGe}_{2} \mathrm{O}_{7}(x$ $=1$ ) is required. Small amount of relevant information is presently known about the properties of the Co-based system. ${ }^{17}$ In the present paper, we report the results of a preliminary magnetic neutron-diffraction study that confirms the previously hypothesized commensurate nature of the ordered state. A subsequent detailed measurement of the spinwave dispersion relation yields the relevant energy scales of magnetic interactions and helps us to explain the observed commensurability of the ground state in $\mathrm{Ba}_{2} \mathrm{CoGe}_{2} \mathrm{O}_{7}$, as well as the mechanism of the doping-induced IC transition in $\mathrm{Ba}_{2}\left(\mathrm{Co}_{x} \mathrm{Cu}_{1-x}\right) \mathrm{Ge}_{2} \mathrm{O}_{7}$.

\section{EXPERIMENT}

To date, the exact crystal structure of $\mathrm{Ba}_{2} \mathrm{CoGe}_{2} \mathrm{O}_{7}$ has not been determined. However, powder data ${ }^{21}$ indicate that the material is very similar to its $\mathrm{Cu}$-based counterpart and is characterized by the $P \overline{4} 2_{1} m$ crystallographic space group. The lattice parameters for $\mathrm{Ba}_{2} \mathrm{CoGe}_{2} \mathrm{O}_{7}$ are $a$ $=b=8.410 \AA$ and $c=5.537 \AA$, as measured at $T=10 \mathrm{~K}$. In each crystallographic unit cell, the magnetic $\mathrm{Co}^{2+}$ ions are 
located at $(0,0,0)$ and $(0.5,0.5,0)$ positions. The NN Co-Co distance is thus along the $(1,1,0)$ direction and equal to $a / \sqrt{2} \approx 5.9 \AA$. For the present study, we utilized two singlecrystal samples prepared using the floating-zone technique. Both crystals were cylindrical, roughly $5 \mathrm{~mm}$ diameter $\times 50 \mathrm{~mm}$ long, with a mosaic spread of about $0.4^{\circ}$.

The first series of experiments was carried out at the HB1 3 -axis spectrometer installed at the High Flux Isotope reactor at Oak Ridge National Laboratory (setup I). Its main purpose was to determine the spin arrangement in the magnetically ordered state. The sample was mounted with the $b$ axis vertical making $(h, 0, l)$ reflections accessible for measurements. Neutrons with a fixed incident energy of $13.5 \mathrm{meV}$ were used in combination with a pyrolytic graphite (PG) monochromator and analyzer, $30^{\prime}-40^{\prime}-20^{\prime}-120^{\prime}$ collimation, and a PG higher-order filter. Sample environment was a closed-cycle refrigerator that allowed measurements at temperatures down to $3.5 \mathrm{~K}$. To isolate the magnetic contribution, integrated intensities were measured in a series of rocking curves at $T$ $=3.5 \mathrm{~K}<T_{\mathrm{N}}$ and $T=10 \mathrm{~K}>T_{\mathrm{N}}$. While using setup I, it became apparent that the study of magnetic excitations could be much better carried out using a cold neutron instrument, the relevant energy scale for $\mathrm{Ba}_{2} \mathrm{CoGe}_{2} \mathrm{O}_{7}$ being about 2 $\mathrm{meV}$. These measurements, which are a central part of the present study, were performed at the TASP 3-axis spectrometer installed at the SINQ spallation source at Paul Scherrer Institut (setup II). Neutrons with a fixed final energy of 5.5 $\mathrm{meV}$ were used with PG monochromator and analyzer, and a PG filter after the sample. The beam collimation was (guide) $-80^{\prime}-80^{\prime}$-(open). The sample was mounted with the $c$ axis vertical, making momentum transfers in the $(h, k, 0)$ reciprocal-space plane accessible for measurement. Spin wave dispersion curves were measured along the $(h, 0,0)$ and $(h, h, 0)$ directions using constant- $Q$ scans in the energy range $0-4 \mathrm{meV}$. The sample environment was a standard "ILL Orange" He-4 flow cryostat, and most of the data were taken at $T=2 \mathrm{~K}$.

\section{RESULTS}

\section{A. An approximate model for the magnetic structure}

In $\mathrm{Ba}_{2} \mathrm{CuGe}_{2} \mathrm{O}_{7}$, magnetic ordering gives rise to incommensurate peaks surrounding the integer $h, k$, and $l$ reciprocal-space points. ${ }^{3}$ In contrast, in $\mathrm{Ba}_{2} \mathrm{CoGe}_{2} \mathrm{O}_{7}$ magnetic Bragg scattering was detected below $T_{\mathrm{N}}=6.7 \mathrm{~K}$ at strictly commensurate positions $h, k$ and $l$-integer. Due to their location, the magnetic reflections, except those on the $(h, 0,0)$ and $(0, k, 0)$ reciprocal-space rods, coincide with nuclear ones. Figure 1 shows the measured temperature dependence of the $(1,0,0)$ peak intensity (setup II). The inset shows rocking curves measured above and below the ordering temperature.

The appreciable residual intensity seen at $T>T_{N}$ at the $(1,0,0)$ forbidden nuclear peak position is due to multiple scattering. In fact, multiple scattering and extinction effects are rather severe in our large samples, that were prepared for inelastic studies, rather than for diffraction work. Performing differential measurements at low temperature and above $T_{N}$,

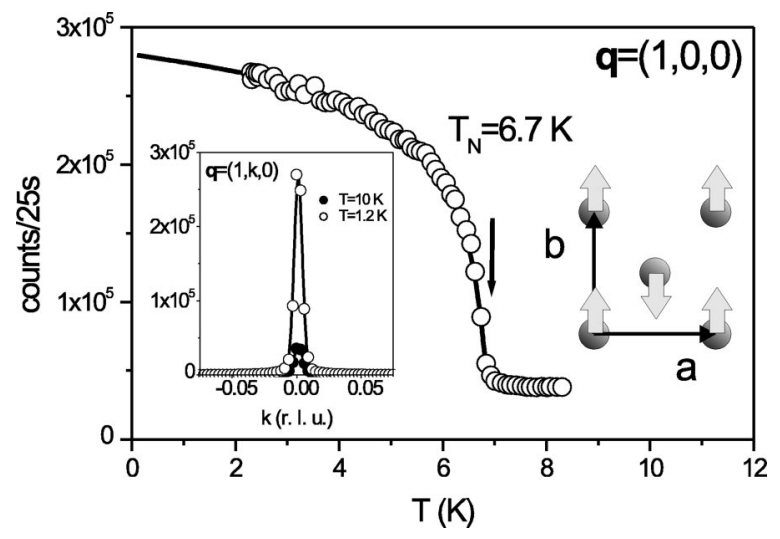

FIG. 1. Measured temperature dependence of the $(1,0,0)$ magnetic Bragg peak intensity in $\mathrm{Ba}_{2} \mathrm{CoGe}_{2} \mathrm{O}_{7}$ (symbols). The solid line is a guide to the eye. Left inset: transverse scans (rocking curves) across the $(1,0,0)$ Bragg reflection measured below (open circles) and above (solid circles) the Néel temperature $T_{N} \approx 6.7 \mathrm{~K}$. The residual intensity seen at $T>T_{\mathrm{N}}$ is of nonmagnetic origin and due to multiple scattering. Right inset: the proposed model for the spin structure of $\mathrm{Ba}_{2} \mathrm{CoGe}_{2} \mathrm{O}_{7}$. All spins are within the $(a, b)$ crystallographic plane.

as described in the preceding section, does not get rid of the problem entirely: multiple scattering involving magnetic Bragg reflections remains. As a result, the data quality is limited by systematic errors. An accurate determination of the spin structure calls for future experiments using dedicated diffraction instruments and much smaller sample. Even at the present stage, however, some important conclusions regarding the overall geometry of the spin arrangement in $\mathrm{Ba}_{2} \mathrm{CoGe}_{2} \mathrm{O}_{7}$ can be drawn from our preliminary data.

It is well known that integrated Bragg intensities measured using a 3-axis spectrometer should be treated with some caution. ${ }^{19}$ In general, one cannot use conventional expressions for the Lorenz factor to extract the structure factors from the measured intensities. ${ }^{20}$ Instead, one has to utilize the full four-dimensional resolution function of a 3-axis spectrometer that can be calculated, for example, using the Cooper-Nathans $^{22,23}$ or Popovici ${ }^{23}$ approximations. By the very definition of the resolution function, it follows that the peak (not integrated) intensity $I_{h k l}^{(\text {peak) }}$ measured at the Bragg position $\mathbf{q}=\mathbf{q}_{h k l} \equiv h \mathbf{a}^{*}+k \mathbf{b}^{*}+l \mathbf{c}^{*}$ is given by

$$
I_{h k l}^{(\text {peak })} \propto I_{h k l} / V_{h k l},
$$

where $I_{h k l} \equiv\left|\hat{q}_{h k l} \times \mathbf{F}_{h k l} \times \hat{q}_{h k l}\right|^{2}$ is the absolute value of the projection of the magnetic structure factor perpendicular to the scattering vector and $V_{h k l}$ is the four-dimensional volume of the resolution ellipsoid at $\mathbf{q}=\mathbf{q}_{h k l}$. The mosaic of the sample, in a Gaussian approximation, is usually explicitly taken into account in the resolution calculation. Nevertheless, in practice, for increased accuracy, one measures a rocking curve for each Bragg peak and determines the integrated intensities $I_{h k l}^{(\omega)}$. An estimate for the peak intensity is then obtained assuming $I_{h k l}^{(\text {peak })} \propto I_{h k l}^{(\omega)} / W_{\omega}$, where $W_{\omega}$ is the measured width of the peak in the rocking scan.

In the present study, we applied this method to analyze 19 nonequivalent magnetic Bragg intensities measured as de- 
TABLE I. Magnetic Bragg intensities measured in $\mathrm{Ba}_{2} \mathrm{CoGe}_{2} \mathrm{O}_{7}$ at $T=3.5 \mathrm{~K}$, after subtracting the background measured at $T$ $=10 \mathrm{~K}$, in comparison to those calculated for the proposed approximate collinear antiferromagnetic structure.

\begin{tabular}{rcccccc}
\hline \hline$h$ & $k$ & $l$ & $I_{\text {calc }}$ & $I_{\text {obs }}$ & $\sigma_{\text {obs }}$ & $I_{\text {obs }}-I_{\text {calc }}$ \\
\hline & & & & & & $\sigma_{\text {obs }}$ \\
\hline-1 & 0 & 0 & 9031 & 8226 & 186 & -4.3 \\
-2 & 0 & 0 & 0 & 100 & 129 & 0.8 \\
-3 & 0 & 0 & 5577 & 5702 & 84 & 1.5 \\
-4 & 0 & 0 & 0 & 1405 & 4013 & 0.4 \\
-5 & 0 & 0 & 2340 & 3954 & 212 & 7.6 \\
0 & 0 & 1 & 0 & -22 & 1323 & -0.0 \\
-1 & 0 & 1 & 13294 & 12187 & 230 & -4.8 \\
-2 & 0 & 1 & 0 & -59 & 223 & -0.3 \\
-3 & 0 & 1 & 5880 & 875 & 1260 & -4.0 \\
-4 & 0 & 1 & 0 & 220 & 301 & 0.7 \\
-5 & 0 & 1 & 2260 & 3257 & 1690 & 0.6 \\
0 & 0 & 2 & 0 & 21 & 106 & 0.2 \\
-2 & 0 & 2 & 0 & 70 & 243 & 0.3 \\
-3 & 0 & 2 & 5018 & 8775 & 242 & 15.5 \\
-4 & 0 & 2 & 0 & -150 & 325 & -0.5 \\
0 & 0 & 3 & 0 & 689 & 3308 & 0.2 \\
-1 & 0 & 3 & 5400 & 5081 & 1897 & -0.2 \\
-2 & 0 & 3 & 0 & 269 & 123 & 2.2 \\
-3 & 0 & 3 & 3134 & 4115 & 6000 & 0.2 \\
\hline \hline
\end{tabular}

scribed in the previous section. The resolution function was calculated in the Cooper-Nathans approximation. The resolution-corrected magnetic intensities $I_{\mathrm{obs}}$ are listed in Table I. While signs of weak ferromagnetism are clearly manifest in the bulk susceptibility curves of Ref. 17, the quality of the present diffraction dataset is not sufficient to analyze the subtle canting of the spin structure typically responsible for this effect. Instead, we choose to analyze the observed intensity pattern using an a priori oversimplified collinear model. The actual spin arrangement in $\mathrm{Ba}_{2} \mathrm{CoGe}_{2} \mathrm{O}_{7}$ is likely to be a canted version of this structure. Note that in $\mathrm{Ba}_{2} \mathrm{CoGe}_{2} \mathrm{O}_{7}$, the magnetic sites are located in high-symmetry positions. As a result, for the proposed model the magnetic unit-cell structure factors can be calculated exactly, without knowing the details of the crystal structure. Given all the assumptions and the systematic experimental errors involved, a reasonably good agreement with experiment can be obtained assuming all spins confined to the $(a, b)$ plane and nearest-neighbor spins aligned antiparallel with each other. The alignment of nearest-neighbor spins along the $c$ direction is "ferromagnetic." Such a spin structure is identical to the one in the commensurate spin-flop phase of $\mathrm{Ba}_{2} \mathrm{CuGe}_{2} \mathrm{O}_{7}$ stabilized by an external magnetic field applied along the $c$ axis. ${ }^{4,5}$ Magnetic Bragg intensities calculated from this model are listed in Table I for direct comparison with experiment. Due to the possibility of antiferromagnetic domains, the spin orientation within the $(a, b)$ plane could not be determined unambiguously. Neither did we measure the actual magnitude of the ordered moment,
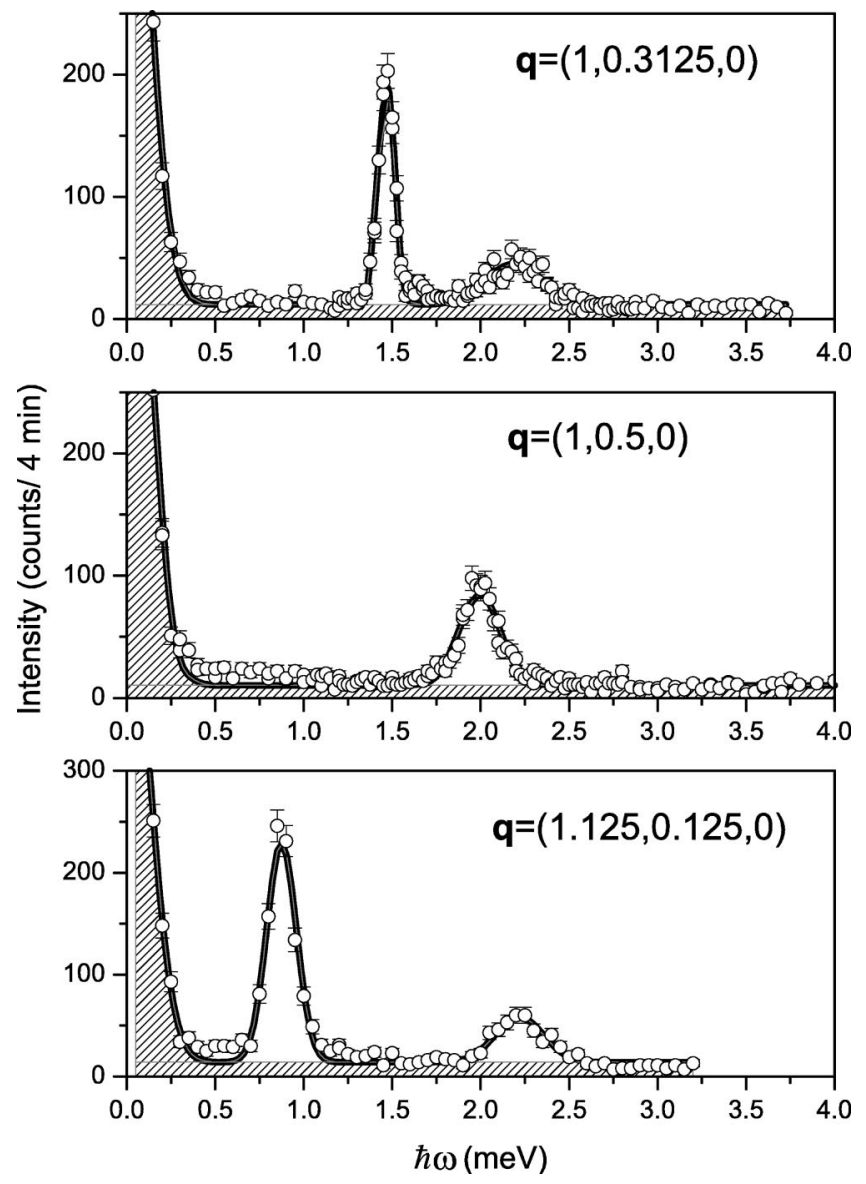

FIG. 2. Typical constant- $Q$ scans measured in $\mathrm{Ba}_{2} \mathrm{CoGe}_{2} \mathrm{O}_{7}$ at $T=2 \mathrm{~K}$. The solid lines are Gaussian fits to the data. Shaded areas represent the background level.

since the crystallographic data needed to bring the measured magnetic intensities to an absolute scale is not currently available for $\mathrm{Ba}_{2} \mathrm{CoGe}_{2} \mathrm{O}_{7}$.

\section{B. Spin waves}

We now turn to discussing the main results of this work, namely, the measured dispersion of spin-wave excitations in $\mathrm{Ba}_{2} \mathrm{CoGe}_{2} \mathrm{O}_{7}$ that we find to be quite different from the one previously seen in $\mathrm{Ba}_{2} \mathrm{CuGe}_{2} \mathrm{O}_{7} \cdot{ }^{7,8}$ Figure 2 shows typical constant $-q$ scans measured in the Co-compound using setup II at $T=2 \mathrm{~K}$. Two distinct sharp excitations are observed. One branch is acoustic in origin, with excitation energy linearly going to zero at the $\mathrm{AF}$ zone center $(1,1,0)$. The second "optical" branch is barely dispersive and is always seen around $2 \mathrm{meV}$ energy transfer. The two spin-wave branches converge at the AF zone boundary $(0.5,0.5,0)$. In all cases, the observed energy width of spin-wave peaks is resolution limited. The apparent variation of peak width seen in Fig. 2 is due to instrumental "focusing" effects. The data were analyzed using Gaussian fits (solid line in Fig. 2). The background (shaded areas) was assumed to be constant with an additional Gaussian component at zero-energy transfer to model incoherent elastic scattering. The dispersion relations along the $(1,1,0)$ and $(1,0,0)$ reciprocal-space directions deduced from these fits are plotted as symbols in Fig. 3. 

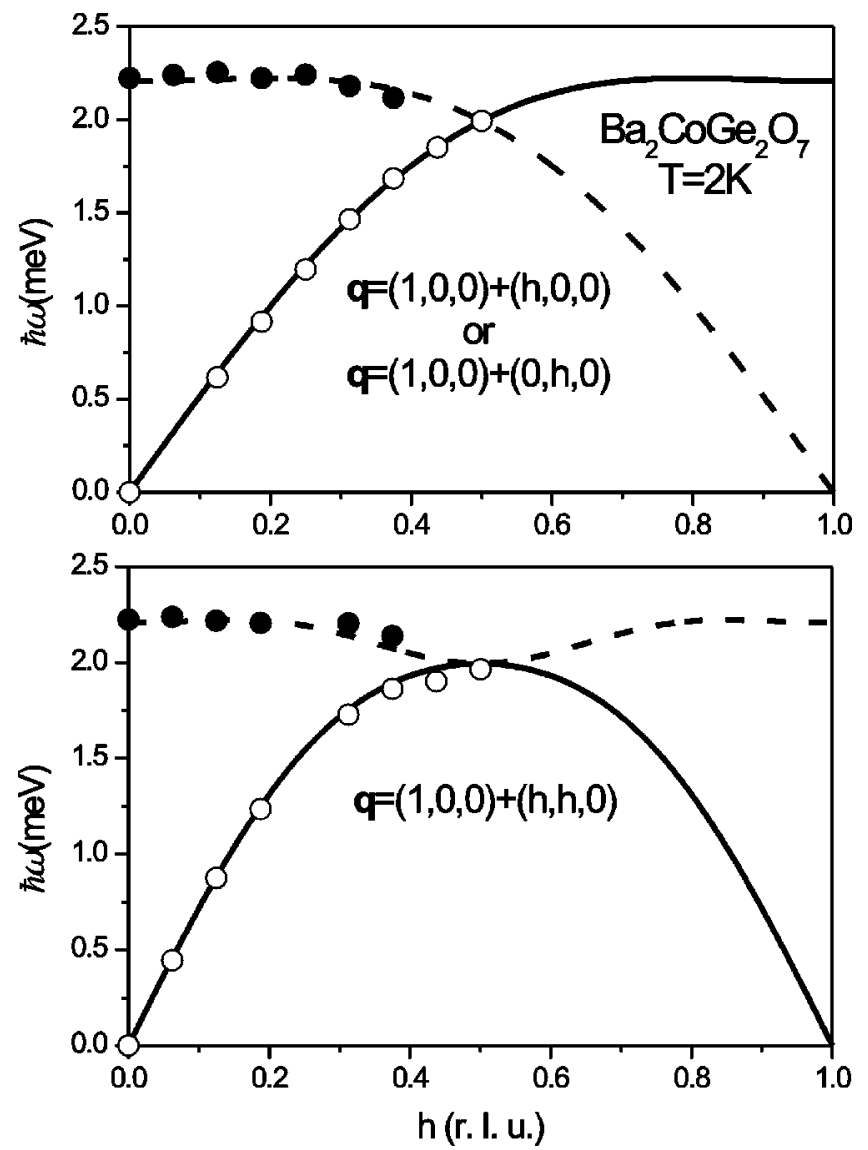

FIG. 3. Dispersion of spin waves along the $(1,0,0)$ and $(1,1,0)$ reciprocal-space directions measured in $\mathrm{Ba}_{2} \mathrm{CoGe}_{2} \mathrm{O}_{7}$ at $T=2 \mathrm{~K}$ (symbols). The solid and dashed lines represent the two spin-wave branches in the model defined by Eq. (2), with dispersion relations given by Eq. (3), and parameters chosen to best fit the data.

\section{Data analysis}

Compared to its $\mathrm{Cu}$-based counterpart, where the magnetic $\mathrm{Cu}^{2+}$ are reasonably well described as true $S=1 / 2$ ions, the magnetism of $\mathrm{Ba}_{2} \mathrm{CoGe}_{2} \mathrm{O}_{7}$ can be very complicated due to the involvement of $\mathrm{Co}^{2+}$. This $S=3 / 2$ ion is often characterized by strong spin-orbit interactions, and the entire set of spin and orbital degrees of freedom should, in principle, be considered, and may give rise to a complex crystal-field level scheme. Fortunately, as follows from our measurements of the magnetic excitations in $\mathrm{Ba}_{2} \mathrm{CoGe}_{2} \mathrm{O}_{7}$, the low-energy physics (at least up to $4 \mathrm{meV}$ energy transfer) can be described in terms of effective spins $S=1 / 2$, associated with the lowest-energy Kramers doublets of the $\mathrm{Co}^{2+}$ ions. Given the structural similarity with $\mathrm{Ba}_{2} \mathrm{CuGe}_{2} \mathrm{O}_{7}$, the effective spin Hamiltonian for $\mathrm{Ba}_{2} \mathrm{CoGe}_{2} \mathrm{O}_{7}$ should also include dominant magnetic interactions between nearest-neighbor sites in the $(a, b)$ plane. However, unlike in the $\mathrm{Ba}_{2} \mathrm{CuGe}_{2} \mathrm{O}_{7}$ case, given the nature of the effective spin variables for $\mathrm{Ba}_{2} \mathrm{CoGe}_{2} \mathrm{O}_{7}$, these interactions can be highly anisotropic. This anisotropy is reflected in the effective spin Hamiltonian:

$$
\hat{\mathcal{H}}=\sum_{m} \sum_{n}^{\tilde{n}}\left[J_{z} S_{m}^{(z)} S_{n}^{(z)}+J_{\perp} S_{m}^{(x)} S_{n}^{(x)}+J_{\perp} S_{m}^{(y)} S_{n}^{(y)}\right]
$$

Here, $m$ and $n$ label the spins on the two antiferromagnetic square sublattices with origins at $(0,0,0)$ and $(0.5,0.5,0)$, respectively, and $\widetilde{\Sigma}$ stands for summation over nearest neighbors. It is important to emphasize that $J_{z}$ and $J_{\perp}$ are introduced as phenomenological coupling constants between effective spin operators and their microscopic meaning remains hidden. Nevertheless, it is these constants that define the behavior of the system at low energies, as described by the effective spin model. Note that interactions along the $c$ axis are not included in Eq. (2). They were not measured directly in this work, and are likely to be ferromagnetic, due to the value of the magnetic ordering vector. The corresponding coupling constant was previously found to be extremely weak in $\mathrm{Ba}_{2} \mathrm{CuGe}_{2} \mathrm{O}_{7}{ }^{3}$ and was assumed to also be small in the Co system. The $c$-axis coupling should thus have no considerable effect on spin-wave dispersion in the $(h, k, 0)$ reciprocal-space plane.

The low-energy excitation spectrum of the effective spin model can be derived from the effective Hamiltonian (2) in the framework of conventional spin-wave theory. The corresponding dispersion relation can be obtained, for example, through a linearized Holstein-Primakoff transformation of effective spin operators in Eq. (2). The resulting spin-wave Hamiltonian is diagonalized by a Fourier-Bogolyubov transformation. This straightforward yet tedious calculation for our particular case yields two spin-wave branches with the following dispersion relations:

$$
\left[\hbar \omega_{\mathbf{q}}^{( \pm)}\right]^{2}=(4 S)^{2}\left(2 J_{\perp} \mp J_{\perp} C_{\mathbf{q}}\right)\left(2 J_{\perp} \pm J_{z} C_{\mathbf{q}}\right),
$$

where

$$
C_{\mathbf{q}} \equiv \cos (\pi h+\pi k)+\cos (\pi h-\pi k) .
$$

Excellent fits to the data are obtained with effective spin $S$ $=1 / 2$ and assuming the effective coupling constants to be $J_{\perp}=0.496(5) \mathrm{meV}$ and $J_{z}=0.192(3) \mathrm{meV}$. Dispersion curves calculated using these parameters are shown in dashed and solid lines in Fig. 3. The main conclusion of this analysis is that the low-energy physics of $\mathrm{Ba}_{2} \mathrm{CoGe}_{2} \mathrm{O}_{7}$ is very well described by a highly anisotropic effective spin model.

\section{DISCUSSION}

The effective spin model allows us to discuss the magnetic structure, phase transitions, and low-energy spin dynamics in $\mathrm{Ba}_{2} \mathrm{CoGe}_{2} \mathrm{O}_{7}$ using the same convenient "spin" terminology as for the $\mathrm{Cu}$-based compound. In particular, all the symmetry-permitted terms in the spin Hamiltonian of $\mathrm{Ba}_{2} \mathrm{CuGe}_{2} \mathrm{O}_{7}$, such as Dzyaloshinskii-Moriya interactions, will have its analogs in the effective spin Hamiltonian of $\mathrm{Ba}_{2} \mathrm{CoGe}_{2} \mathrm{O}_{7}$. Unlike in $\mathrm{Ba}_{2} \mathrm{CuGe}_{2} \mathrm{O}_{7}$, however, the strong anisotropy effects in the Co material will push all (effective) spins into the $(a, b)$ crystallographic plane. This effect is similar to that of a magnetic field applied along the $c$ axis that favors an $(a, b)$-planar state in $\mathrm{Ba}_{2} \mathrm{CuGe}_{2} \mathrm{O}_{7} \cdot{ }^{7,8}$ Since the helimagnet-forming uniform components of the Dzyaloshinskii vector $\mathbf{D}_{x y}$ is itself in the $(a, b)$ plane, forcing them makes the corresponding triple-product $\mathbf{D}_{x y}\left(\mathbf{S}_{m} \times \mathbf{S}_{n}\right)$ 
vanish. Only the non-helimagnet-forming sign-alternating $z$-axis component of $\mathbf{D}$ remains relevant. As a result, the magnetic structure may be slightly canted (weak ferromagnetism), but is, nevertheless, commensurate. In this $\mathrm{Ba}_{2} \mathrm{CoGe}_{2} \mathrm{O}_{7}$ greatly resembles another square-lattice weak ferromagnet, namely, $\mathrm{K}_{2} \mathrm{~V}_{3} \mathrm{O}_{8}$ system. $^{16}$

It is important to stress that easy-plane anisotropy was previously detected in $\mathrm{Ba}_{2} \mathrm{CuGe}_{2} \mathrm{O}_{7}$ as well. However, in this material the effect is entirely due to Kaplan interactions, ${ }^{7,8}$ which are very weak and have an energy scale of $D^{2} / J \sim 3 \times 10^{-2} J$. On a square lattice Kaplan interactions happen to be just strong enough to distort the helical structure, but not to fully destroy incommensurability. ${ }^{7}$ In contrast, as follows from the present study, easy-plane effective spin anisotropy in $\mathrm{Ba}_{2} \mathrm{CoGe}_{2} \mathrm{O}_{7}$ is much stronger, of the order of the effective $J$ itself. Its magnitude is well beyond the critical value needed to destroy the incommensurate helimagnetic state.

The results discussed above allows us to speculate about the IC transition in $\mathrm{Ba}_{2}\left(\mathrm{Co}_{x} \mathrm{Cu}_{1-x}\right) \mathrm{Ge}_{2} \mathrm{O}_{7}$ that occurs with increasing Co-concentration $x$. Each Co-impurity strongly "pins" the original spiral at the impurity site, firmly confining the corresponding (effective) spin to the $(a, b)$ plane. Helimagnetic correlations are totally destroyed when the characteristic distance between such strong-pinning locations becomes comparable with the period of the unperturbed spiral, which in $\mathrm{Ba}_{2} \mathrm{CuGe}_{2} \mathrm{O}_{7}$ is roughly 40 nearest-neighbor bonds. ${ }^{3}$ This suggests a critical concentration of about $x \approx 2 \%$, in reasonable agreement with bulk magnetization data of Ref. 17.

\section{CONCLUSION}

To summarize, the magnetism of $\mathrm{Ba}_{2} \mathrm{CoGe}_{2} \mathrm{O}_{7}$ can be described in terms of an effective spin model with strong easyplane anisotropy. Such behavior is manifest in the measured spin-wave excitation spectrum and compatible with preliminary diffraction measurements. The commensurate nature of the ground state, now directly confirmed experimentally, is primarily due to the strong anisotropy effect, and not to a dominant staggered component of the Dzyaloshinskii interactions. As a result, the destruction of helimagnetism in $\mathrm{Ba}_{2}\left(\mathrm{Co}_{x} \mathrm{Cu}_{1-x}\right) \mathrm{Ge}_{2} \mathrm{O}_{7}$ occurs very rapidly with increasing Co concentration, as soon as the mean distance between impurities becomes comparable to the period of the spin spiral.

\section{ACKNOWLEDGMENTS}

This work was partially performed at the spallation neutron source SINQ, Paul Scherrer Institut, Villigen, Switzerland. This work was supported in part by the Grant-in-Aid for COE Research "SCP coupled system" of the Ministry of Education, Culture, Sports, Science and Technology, Japan. Work at ORNL and BNL was carried out under U.S. DOE Contracts Nos. DE-AC05-00OR22725 and DE-AC0298CH10886, respectively.
*Email address: zheludevai@ ornl.gov

${ }^{\dagger}$ Present address: Condensed Matter Sciences Division, Oak Ridge National Laboratory, Oak Ridge, TN 37831-6393, USA.

${ }^{1}$ Y. Ishikawa, K. Tajima, D. Bloch, and M. Roth, Solid State Commun. 19, 525 (1976); Y. Ishikawa, T. Komatsubara, and D. Bloch, Physica B 86-88, 401 (1977); Y. Ishikaya, G. Shirane, J. Tarvin, and M. Kohgi, Phys. Rev. B 16, 4956 (1977); P. Bak and H.M. Jensen, J. Phys. C 13, L881 (1980).

${ }^{2}$ B. Lebech, J. Bernhard, and T. Flertoft, J. Phys.: Condens. Matter 1, 6105 (1989).

${ }^{3}$ A. Zheludev, G. Shirane, Y. Sasago, N. Koide, and K. Uchinokura, Phys. Rev. B 54, 15163 (1996); 55, 11 879(E) (1996).

${ }^{4}$ A. Zheludev, S. Maslov, G. Shirane, Y. Sasago, N. Koide, and K. Uchinokura, Phys. Rev. Lett. 78, 4857 (1997).

${ }^{5}$ A. Zheludev, S. Maslov, G. Shirane, Y. Sasago, N. Koide, and K. Uchinokura, Phys. Rev. B 57, 2968 (1998).

${ }^{6}$ A. Zheludev, S. Maslov, G. Shirane, Y. Sasago, N. Koide, K. Uchinokura, D.A. Tennant, and S.E. Nagler, Phys. Rev. B 56, 14006 (1997).

${ }^{7}$ A. Zheludev, S. Maslov, I. Tsukada, I. Zaliznyak, L.P. Regnault, T. Masuda, K. Uchinokura, R. Erwin, and G. Shirane, Phys. Rev. Lett. 81, 5410 (1998).

${ }^{8}$ A. Zheludev, S. Maslov, G. Shirane, I. Tsukada, T. Masuda, K. Uchinokura, I. Zaliznyak, R. Erwin, and L.P. Regnault, Phys. Rev. B 59, 11432 (1999).

${ }^{9}$ T. Moriya, Phys. Rev. 120, 91 (1960).

${ }^{10}$ T.A. Kaplan, Z. Phys. B: Condens. Matter 49, 313 (1983).

${ }^{11}$ L. Shekhtman, O. Entin-Wohlman, and A. Aharony, Phys. Rev.
Lett. 69, 836 (1992).

${ }^{12}$ L. Shekhtman, A. Aharony, and O. Entin-Wohlman, Phys. Rev. B 47, 174 (1993).

${ }^{13}$ O. Entin-Wohlman, A. Aharony, and L. Shekhtman, Phys. Rev. B 50, 3068 (1994).

${ }^{14}$ J. Chovan, N. Papanicolaou, and S. Komineas, Phys. Rev. B 65, 064433 (2002).

${ }^{15}$ A.N. Bogdanov, U.K. Rössler, M. Wolf, and K.-H. Müller, cond-mat/0206291 (unpublished).

${ }^{16}$ M.D. Lumsden, B.C. Sales, D. Mandrus, S.E. Nagler, and J.R. Thompson, Phys. Rev. Lett. 86, 159 (2001).

${ }^{17}$ T. Sato, T. Masuda and K. Uchinokura, in Proceedings of the $23 \mathrm{rd}$ International Conference on Low Temperature Physics [Physica B (to be published)].

${ }^{18}$ I.E. Dzyaloshinskii, Zh. Eksp. Teor. Fiz. 46, 1420 (1964) [Sov. Phys. JETP 19, 960 (1964)].

${ }^{19}$ G. Shirane, S.M. Shapiro, and J. Tranquada, Neutron Scattering with a Triple-Axis Spectrometer (Cambridge University Press, Cambridge, 2002), Chap. 7.2.

${ }^{20}$ M. Iizumi, Jpn. J. Appl. Phys. 12, 167 (1973).

${ }^{21}$ H.F. McMurdie, F. Howard, M.C. Morris, E.H. Evans, B. Paretzkin, W. Wong-Ng, L. Ettinger, and C.R. Hubbard, Powder Diffr. 1, 64 (1986).

${ }^{22}$ M.J. Cooper and R. Nathans, Acta Crystallogr. 23, 357 (1967); N.J. Chesser and J.D. Axe, Acta Crystallogr., Sect. A: Cryst. Phys., Diffr., Theor. Gen. Crystallogr. A29, 160 (1973).

${ }^{23}$ M. Popovici, Acta Crystallogr., Sect. A: Cryst. Phys., Diffr., Theor. Gen. Crystallogr. A31, 507 (1975). 